\title{
Strongyle infections in sheep and goats under the traditional husbandry system in Peninsular Malaysia.
}

\begin{abstract}
Faecal egg counts were used to study patterns of trichostrongyle infections in sheep and goats according to season, age, pregnancy and lactation on traditional farms in west Malaysia. Haemonchus contortus and Trichostrongylus spp. were the most important strongyles in sheep and in goats, H. contortus, Trichostrongylus spp. and Oesophagostomum spp. were most prevalent. The faecal egg counts of sheep and goats were apparently not influenced by the small seasonal climatic variations. Strongyle infections were acquired at an earlier age in sheep than in goats. Mean faecal egg counts decreased from the age of 8 months onwards in sheep while in goats this occurred from 12-18 months onwards. A periparturient rise in strongyle egg counts was observed in both animal species. Haemonchus contortus was mainly responsible for this rise in faecal egg counts. The results are discussed with reference to control of gastrointestinal strongyle infections in sheep and goats.
\end{abstract}

Keyword: Sheep-Nematoda, Goat, Epidemiology-Nematoda, Periparturient rise 